Documents pour l'histoire des techniques

Nouvelle série

20 | 2e semestre 2011

L'expérimentation « en plein air » ou " grandeur nature ": Une pratique scientifique au service de l'action (XIXe-XXe siècles)

\title{
Arthur Morin et les expériences hydrodynamiques à la Commission dite des principes du tir
} (1836-1841)

Arthur Morin's hydrodynamic experiments at the so called «Commission des

principes du tir » (1836-1841)

\section{Claudine Fontanon}

\section{(2) OpenEdition}

Journals

Édition électronique

URL : http://journals.openedition.org/dht/1650

DOI : $10.4000 /$ dht. 1650

ISSN : 1775-4194

Éditeur :

Centre d'histoire des techniques et de l'environnement du Cnam (CDHTE-Cnam), Société des élèves du CDHTE-Cnam

Édition imprimée

Date de publication : 1 décembre 2011

Pagination : 59-70

ISBN : 978-2-9530779-7-1

ISSN : 0417-8726

\section{Référence électronique}

Claudine Fontanon, «Arthur Morin et les expériences hydrodynamiques à la Commission dite des principes du tir (1836-1841) », Documents pour l'histoire des techniques [En ligne], 20 | 2e semestre 2011, mis en ligne le 25 septembre 2012, consulté le 08 septembre 2020. URL : http:// journals.openedition.org/dht/1650; DOI : https://doi.org/10.4000/dht.1650 


\title{
Arthur Morin et les expériences hydro- dynamiques à la Commission dite des principes du tir (1836-1841)
}

\author{
Claudine Fontanon \\ EHESS-Centre Alexandre Koyré UMR 8560
}

\begin{abstract}
Résumé
Au début des années 1830, le ministère de la Guerre décide d'améliorer la formation des élèves des écoles d'artillerie en proposant un programme de recherches appliquées dans le domaine de la balistique. C'est dans ce contexte qu'Arthur Morin et deux de ses collègues artilleurs, Guillaume Poiberte et Isidore Didion, proposent d'étudier la résistance des différents milieux à la pénétration des projectiles. Ces derniers vont mettre au point une série d'expériences en plein air sur les lois de la résistance de l'air et de l'eau à la pénétration des projectiles. Arthur Morin qui comme nombre de polyłechniciens est préoccupés par l'amélioration des performances de l'industrie et des transports va largement dépasser l'objectif et le cadre temporel assigné par la Commission dite « des principes du tir » en se livrant à des expériences sur la résistance de l'eau à l'avancement des corps immergés et flottants qui s'appliquent aux roves des bateaux à vapeur et à la navigation fluviale. C'est à ces expériences d'hydrodynamique menées par Arthur Morin en plein air de 1836 à 1841 avec l'assentiment du ministère de la Guerre et en marge des questions de balistique, que cet article est consacré.
\end{abstract}

Résumés et mots clés en anglais sont regroupés en fin de volume, accompagnés des mots clés français

Il y a près de vingt ans, une équipe de chercheurs s'est attelée sous la direction de Louis Bergeron, alors directeur du CRH/EHESS, à une recherche de longue haleine sur les moteurs hydrauliques en France au XIXe siècle'. Cette recherche renvoyait en amont du niveau de l'objet construit et de son utilisateur vers celui du savoir scientifique et de la recherche théorique et de la formulation mathématique dans le domaine de l'hydrodynamique : "La réflexion comparative entre la France d'une part, l'Angleterre et la Nouvelle Angleterre de l'autre (...) incitait à travailler sur le rôle dans cette affaire du nouveau savoir de l'ingénieur à l'aube du XXe siècle, sur l'importance des méthodes associant l'expérimentation, la vérification sur le terrain à la formulation mathématique. C'est ainsi qu'apparut sous une nouvelle lumière le rôle joué par des personnages aussi différents par leur formation ou leur carrière que Jean Victor Poncelet, Arthur Morin ou Sagebien, en tant que chercheurs, inventeurs et diffuseurs de l'invention (...) $\|^{2}$.

\footnotetext{
1 « Le moteur hydraulique en France au XIXe siècle : concepteurs, inventeurs et constructeurs 11, Cahiers d'histoire et de philosophie des sciences, nouvelle série, n² 29, 1990, 318 p.

2 Louis Bergeron, "Avant-propos ", dans Le moteur hydraulique, op. cit., p.III.
}

Poncelet réunit autour de lui une pléiade de jeunes ingénieurs, dont Arthur Morin, J. Lesbos, Guillaume Piobert ou Isidore Didion, qui s'inspiraient de ses méthodes, poursuivirent ses travaux dans le domaine de l'hydraulique. «Ceux-ci passés par l'Ecole polyłechnique et par l'École du génie et de l'artillerie de Metz possédaient une solide formation scientifique et se considéraient comme ingénieurs avant d'être des militaires $\|^{3}$.

C'est dans le cadre de cette recherche collective que Bruno Belhoste associé à Jean-François Belhoste et à Lovis Lemaître analysait la théorie des roves hydrauliques ainsi que l'activité de Jean-Victor Poncelet dans le domaine des roves et turbines hydrauliques ${ }^{4}$ et que nous-mêmes sortions de l'oubli l'ingénieur militaire Arthur Morin, expérimentateur hors pair et médiateur des nouveaux savoirs théoriques et pratiques dans ce domaine ${ }^{5}$.

\footnotetext{
3 Bruno Belhoste, "Jean-Victor Poncelet, les ingénieurs militaires et les roves et moteurs hydrauliques ", dans " Le moteur hydraulique ॥, op. cit., pp. 33-89.

4 Bruno Belhoste, "La théorie des machines et les roves hydrauliques ", dans Le moteur hydraulique, op. cit., pp. 1-32 ; id., « Jean-Victor Poncelet », op. cit.

5 Claudine Fontanon, "Arthur Morin (1795-1880).
} 
La balistique intérieure est la partie de la physique qui étudie le mouvement d'un projectile à l'intérieur de la bouche à feu. Pour l'artilleur Arthur Morin, la détermination des pressions exercées par les gaz dans l'âme des bouches à feu, la loi des vitesses successives communiquées aux projectiles constituent la balistique intérieure. Guillaume Piobert fut le premier à établir une théorie mathématique de ces effets complexes basée sur de nombreuses expériences exécutées à Metz entre 1834 et 1838 pour la Commission des principes du tir.

La balistique extérieure est la branche de la physique qui étudie le vol des projectiles sans propulsion interne. Sa principale application est l'étude des trajectoires des obus ou des balles tirés par des armes à feu après qu'ils aient quitté le canon de l'arme.

Une somme considérable de rapports manuscrits et imprimés furent consultés au cours de cette recherche mais certains aspects furent volontairement laissés de côté : leurs activités de recherche menées au titre de leur fonctions militaires. Ceci est particulièrement vrai pour l'artilleur Arthur Morin, dont tout un pan de l'œuvre expérimentale, effectuée au sein d'une commission fondée en 1833 et baptisée Commission des principes du tir, n'a pas été analysé. C'est à ces recherches militaires que nous consacrerons cet article car elles sont exemplaires d'une démarche scientifique largement ignorée des historiens des sciences : l'expérimentation "en plein air " et "en vraie grandeur ", une pratique d'ingénieurs au XIXe siècle. E† c'est plus particulièrement la question de la résistance de l'eau à l'avancement des corps qui sera ici examinée.

\section{Le cadre institutionnel}

Selon J. Challéat, ingénieur militaire et historien de l'artillerie en France au XIXe siècle, les années 18301850 sont celles d'une profonde réorganisation au sein de l'artillerie et de renouvellement des études et recherches lancées par le Comité de l'artillerie. Cette institution fondée en 1795 a pour fonction de veiller à la qualité des enseignements donnés dans les écoles d'application (il en existe huit en 1830), de proposer des programmes d'études et d'expériences pour améliorer le matériel d'artillerie ou le fonctionnement des arsenaux. Ces programmes sont soumis aux écoles d'artillerie chargées de créer des commission temporaires ad hoc dotées de budgets importants pour leur organisation et la réalisation des programmes élaborés par les équipes d'ingénieurs militaires désignés dans chaque école ${ }^{6}$.

Un ingénieur militaire au service de l'industrialisation "), dans Le moteur hydraulique, op. cit., pp. 90-118.

6 Jules Challéat, L'artillerie de terre en France pendant un siècle. Histoire technique, 1816-1919, Paris, 1933, vol.1 (Les écoles d'artillerie sont alors implantées à Besançon, Douai,
Au début des années 1830, le Ministère de la Guerre décide d'augmenter la productivité de ses arsenaux et d'améliorer la formation des élèves des écoles d'artillerie en lançant des programmes de recherches appliquées dans le domaine des moteurs hydrauliques et de la balistique. A cette époque, les élèves-artilleurs ne disposaient pour leur formation que d'un aide-mémoire ancien publié par le général Gassendi à la fin des années 1810 et qui en était à sa cinquième édition en 1830. Le Comité de l'Artillerie du ministère de la Guerre entreprend en 1833 d'actualiser cet aide-mémoire en soumettant aux huit écoles d'artillerie un vaste programme de recherche sur les notions fondamentales de balistique intérieure et extérieure (encadré ci-dessus) ${ }^{7}$.

Le Comité précise que chaque commission " donnera un rapport faisant connaître les sources auxquelles elle aura puisé, la marche et les procédés divers qu'elle aura suivis $11^{8}$. A Metz la Commission des principes du tir est créée le 29 mai 1833 ; y sont affectés les capitaines d'artillerie Guillaume Piobert et Arthur Morin bientôt rejoint par le capitaine Isidore Didion. Elle est chargée conjointement avec l'École de La Fère de la question des tirs à boulets des pièces de campagne, des tirs à mitraille et des tirs de mortier. Mais à Metz, les trois ingénieurs militaires demandent à étudier préalablement la question non élucidée de

La Fère, Metz, Rennes, Strasbourg, Toulouse et Vincennes). Le rôle de ces écoles est important car elles assurent l'instruction des personnels du corps, des troupes stationnées et exécutent les expériences sur ordre du Comité de l'Artillerie. 7 Le Comité de l'artillerie était un organe consultatif du Ministère de la Guerre. Créé par un arrêté de la Convention du 7 mai 1795, il siégeait dans un ancien couvent des Dominicains-Jacobins de Saint Thomas d'Aquin sur la place du même nom dans le $7^{\mathrm{e}}$ arrondissement de Paris. Son rôle était d'étudier et de discuter les questions que lui adressait la Direction de l'artillerie. Mais comme il ne disposait pas de commission d'expériences, il s'adressait soit aux arsenaux de construction, soit aux écoles militaires (huit au total réparties sur le territoire). 8 Jules Challéat, Histoire technique ... op. cit. , p. 77. 
la vitesse initiale des projectiles et l'étude des lois de la résistance des différents milieux à la pénétration des projectiles, le programme dépassant largement le cadre initial proposé par le Comité de l'Artillerie'.

L'École du Génie et de l'Artillerie de Metz était entrée en 1825 dans une période florissante avec la nomination du géomètre Jean-Victor Poncelet au cours des machines (1825), du capitaine Guillaume Piobert au cours d'artillerie (1831) et du célèbre géomètre Poisson examinateur des élèves de l'artillerie, et avec la présence de brillants officiers comme Arthur Morin, Jules Lesbros ou Isidore Didion ${ }^{10}$. Cette communauté s'enrichit en 1832 de la présence du général Valée, nommé directeur du Service des poudres qui soutiendra l'activité de la Commission des principes du tir et proposera au ministre de la Guerre de rendre celle-ci permanente. Les trois artilleurs vont déployer une intense activité expérimentale de terrain et produire pas moins de onze rapports manuscrits entre 1834 et $1847^{11}$. Tout en activant les travaux de toutes les commissions de l'aide-mémoire, le Comité suit particulièrement les travaux entrepris à Metz et les fait poursuivre par autorisation ministérielle après la publication de l'aide-mémoire en $1836^{12}$. Dans un rapport de 1839, le Comité signalait au ministre de la Guerre l'importance des résultats déjà obtenus par l'équipe de Metz et de ceux qu'elle poursuivait. II demandait donc que la Commission des principes du tir soit autorisée à continuer les expériences « jusqu'à ce qu'elle ait complètement traité les questions qu'elle s'était proposées $11^{13}$. En fait, les expériences furent menées jusqu'en 1850 soit

9 Patrice Bret, « La recherche appliquée et la recherche expérimentale à l'École de Metz ॥, dans Bruno Belhoste et Antoine Picon dir., L'école d'application de l'artillerie et du génie de Metz (1802-1870). Enseignement et recherches, Actes de la journée d'étude du 2 novembre 1995, Ministère de la Culture, 1996, p. 55 (l'auteur énumère les questions de balistique posées par le comité à la Commission des principes du tir).

10 Bruno Belhoste et Antoine Picon dir , L'école d'application ... op. cit. et notamment Bruno Belhoste et Antoine Picon, "Les caractères généraux de l'enseignement à l'école de l'artillerie et du génie de Metz ॥, pp.18-29.

11 Service Historique de l'Armée de Terre [SHAT] : 2W 296 et 297. La liste des rapports a été établie par Jules Challéat, Histoire technique ... op. cit., p.78.

12 SHAT/ 2W 96 : " La commission des principes du tir offre toutes les garanties désirables de zèle, dec apacité et de réserve dans l'emploi des moyens qui lui sont fournis. Elle a déjà obtenu des résultats auxquels on a reconnu la plus grande valeur soit pour améliorer la pratique soit pour éclairer la théorie. Dans l'intérêt du service il est important que les travaux continuent... " , IIle rapport de la commission, juillet 1836.

13 Jules Challéat, Histoire technique ..., op.cit. p. 77 quinze ans après la publication de la première édition de l'aide-mémoire en 1836'14.

\section{Les acteurs}

Guillaume Piobert (1793-1871), Arthur Morin (17951880) et Isidore Didion (1798-1889) désignés en 1833 comme rapporteurs de la Commission des principes du tir appartiennent à cette génération née sous la Révolution et passée par l'École polytechnique où ils reçoivent une formation scientifique de grande qualité ${ }^{15}$. Au début des années 1830, leur formation militaire est achevée après leur passage comme élèves-ingénieurs à l'Ecole du génie et de l'artillerie de Metz et après avoir participé à diverses campagnes militaires. Distingués par le Comité de l'Artillerie pour leurs qualités, ils sont affectés à l'École de Metz, Morin comme assistant de Poncelet au cours de Machines ${ }^{16}$, Piobert en 1831 comme professeur d'Artillerie où Didion lui succède en 1837.

Guillaume Piobert est né à Lyon le 23 novembre 1793 dans un milieu modeste. Mais il sait, d'après son biographe Arthur Morin ${ }^{17}$, s'imposer des privations pour s'instruire et payer un professeur de mathématiques qui reconnait rapidement ses rares facultés et le prépare au concours d'entrée de l'École polytechnique où il est reçut en 1813 à l'âge de 20 ans. En 1815, il est envoyé à l'École de Metz comme sous lieutenant et en sort premier de sa promotion. Envoyé à Toulouse dans une compagnie d'ouvriers artilleurs, il effectue ses premières recherches avec Tardy sur l'effet utile des turbines et imagine en 1820 un appareil de mesure identique au frein de Prony qui n'a encore fait l'objet d'aucune description. Une mission d'étude en Angleterre lui vaut en 1828 d'être nommé chevalier de l'ordre royal de la Légion d'honneur. En 1831, le capitaine Piobert est nommé professeur au cours d'artillerie pour concevoir un corps de doctrine pour l'artillerie englobant ses

14 Aide-mémoire à l'usage des officiers d'artillerie, BergerLevrault, 1836 ; $2^{\mathrm{e}}$ édition, 1844 ; $3^{\mathrm{e} e}$ édition, 1856 (ces ouvrages conservés aux archives du Service historique de la Défense [SHD] n'ont pu être consultés en raison de travaux effectués dans les réserves).

15 Bruno Belhoste, Amy Dahan, Antoine Picon, La formation polytechnicienne, 1794-1994, Paris, Dunod, 1994.

16 Konstantinos Chatzis, « Jean-Victor Poncelet et la science des machines à l'École de Metz :1825-1870", dans B. Belhoste et A. Picon dir.,L'École d'application... op.cit., pp. 32-40.

17 Arthur Morin, « Notice sur le général Piobert, lue dans la séance annuelle des cinq académies le mercredi 25 novembre 1871 ", Mémoires de l'Académie des sciences de l'Institut de France, tome XXXVIII, Paris Firmin Didot, p. LXVII-CVI. 
recherches et travaux sur l'effet des poudres ${ }^{18}$. Lorsqu'en 1833 la Commission des principes du tir est créée à Metz, il en devient avec Morin et Didion l'un des membres rapporteurs et y œuvre de 1835 à 1837, travaillant notamment sur un élément essentiel de la balistique - la détermination des vitesses initiale des projectiles - et conçoit à l'occasion de nouveaux pendules balistiques ${ }^{19}$. La partie la plus intéressante de ses expériences concerne les recherches sur la loi de la résistance des fluides à l'avancement des projectiles, question complexe et non résolve mise au concours par l'Académie des sciences, pour le prix des sciences physiques qu'il reçoit en 1836. ॥ conduira également les recherches sur la résistance de l'air aux petites et grandes vitesses de 1835 à 1838 et proposera une formule dont Didion vérifiera ultérieurement l'exactitude et qui sera utile au calcul des trajectoires des projectiles aux différentes vitesses et sous diverses inclinaisons. D'après Morin, c'est Piobert qui conçoit un mode de représentation graphique pour traduire les phénomènes complexes en jeu en balistique intérieure et extérieure. Son activité au sein de la Commision des principes du tir a été celle d'un inventeur et d'un théoricien.

En 1837, Piobert est remplacé au cours d'artillerie par Isidore Didion et est finalement élu en 1840 à l'Académie des sciences où Poncelet l'a précédé en $1835^{20}$. Selon Arthur Morin, "son ami et frère d'armes ", Piobert aimait peu parler, encore molns écrire, mais il dut publier un traité d'artillerie théorique et pratique sur l'ensemble de ses travaux qui devint la base de l'enseignement de la plupart des écoles militaires du monde ${ }^{21}$.

Isidore Didion (1798-1878) est né à Thionville dans une famille nombreuse dont le père est négociant. Dès le collège, il montre des dispositions remarquables pour les mathématiques et, est envoyé à Pont-à-Mousson où l'abbé Lalliet, un professeur de renom, le prépare au concours d'entrée à l'École

18 P. Bret, « La recherche appliquée et la recherche expérimentale à l'École de Metz», op. cit., pp. 54-55.

19 « Expériences exécutées à Metz dans les années 183637-38-39 et 1842 pour déterminer au moyen des pendules balistiques les vitesses initiales imprimées aux projectiles par la poudre ordinaire de guerre dans les bouches à feu de l'artillerie française II, VIII' rapport à la Commission des principes du tir de l'École de Metz, Paris 1852

20 CR/AS, tome x p. 504 et 528. Guillaume Piobert est élu avec 50 suffrages contre un seul à Arthur Morin qui s'est également présenté en janvier 1840.

21 Guillaume Piobert, Traité d'artillerie théorique et pratique : partie théorique et expérimentale, Paris imp. Bachelier, 1847 ; 2e édition, Mallet et Bachelet, 1860, 96 p. polytechnique. II y entre en 1817 à 19 ans, en sort sous-lieutenant d'artillerie en 1819 et achève sa formation pendant quatre ans à l'École du Génie et de l'Artillerie de Metz. En 1825, lieutenant de première classe, il est détaché à l'École centrale de pyrotechnie nouvellement créée. Reçu à l'Académie de Metz en 1827, il y rencontre «une pléiade d'officiers remarquables 1), dont Piobert, Morin et Bergery ${ }^{22}$. Après quatre ans passés à la manufacture d'armes de la ville, il intégre l'État-major de l'École de Metz où Piobert vient de créer le cours d'artillerie. Lorsqu'il le remplace en 1837, Didion poursuit l'œuvre ébauchée par le savant artilleur en balistique. Expert auprès du Comité de l'Artillerie pour les questions scientifiques, Didion est nommé examinateur d'entrée à l'École polytechnique. Mais c'est en 1873 seulement qu'il se voit décerner le titre de membre correspondant de l'Académie des sciences (section mécanique) sur un rapport du général Morin.

Nous ne retiendrons de ses travaux scientifiques que ceux que réalisés au sein de la Commission des principes du tir où il est appelé en 1835 aux côtés des capitaines Piobert et de Morin. Au départ de Piobert, il prend seul en 1838 la direction des travaux de la Commission. Son œuvre majeure concerne l'établissement des lois de la résistance de l'air au mouvement des projectiles même s'il co-signe le rapport d'expériences avec Morin et Piobert ${ }^{23}$. Ils reçoivent tous trois en 1839 le grand prix de mathématiques de l'Académie des sciences. Toujours avec Morin et Piobert, Didion avait réalisé en 1835 des expériences de terrain sur la pénétration des projectiles dans l'eau et dans différents milieux ${ }^{24}$, et en 1836 une série d'expériences sur la vitesse initiale imprimée aux projectiles par la poudre de guerre (balistique intérieure) avec un pendule balistique conçu par Piobert et Morin. Didion continue ensuite seul les expériences en 1838, 1839 et 1842. Et l'on ne

22 Colonel Virlet, « Notice sur la vie et les travaux du général Didion, membre honoraire de l'Académie de Metz ", Annales de l'Académie de Metz, séance annuelle, pp. 41-73. 23 Mémoire sur la résistance des liquides et des fluides élastiques au mouvement des corps de diverses formes, présenté pour le concours du grand prix de mathématiques, par MM. Piobert, Morin et Didion, officiers d'artillerie, $1^{\text {er juillet }}$ 1838. Ce mémoire est inséré au Mémorial de l'Artillerie, $n^{\circ} 5$ (1842) et $n^{\circ} 7$ (1852).

24 « Rapport sur un mémoire de Piobert et Morin, capitaines d'artillerie, concernant les expériences faites à Metz en 1834 sur la pénétration des projectiles dans différents milieux résistants et sur la rupture des corps par le choc ॥, Mémoires de l'Académie royale des sciences de l'Institut de France, 1836, t. xv, Dupin, Navier, Poncelet rapporteurs. 
saurait conclure cette courte biographie du général Didion sans mentionner que ce fut le général Morin qui présenta à l'Académie des sciences la notice sur les travaux scientifiques du général Didion à l'occasion de sa candidature tardive au titre de correspondant.

Pour clore ce chapitre des biographies, nous évoquerons le parcours aujourd'hui bien balisé d'Arthur Morin ${ }^{25}$, troisième homme de l'équipe de la Commission des principes du tir dont les expériences d'hydrodynamique en plein air seront analysées au chapitre suivant.

Arthur-Jules Morin est né à Paris le 17 octobre 1795 dans un milieu aisé. II est envoyé au collège Sainte-Barbe où il effectue ses études secondaires ainsi que la préparation au concours d'entrée de l'École polytechnique qu'il intègre à l'âge de 18 $a^{26}{ }^{26}$. II en sort en 1817, second de sa promotion, et se voit affecté à l'Ecole du génie et de l'artillerie de Metz comme élève artilleur. En 1825, il se trouve en garnison à Metz lorsque Jean-Victor Poncelet est chargé d'organiser le cours des machines à l'école d'application et délègue au capitaine Morin, devenu son collaborateur, des recherches expérimentales sur le rendement des machines et des moteurs hydrauliques. Le jeune officier s'acquitte de cette mission avec talent au point d'être remarqué par le Comité de l'Artillerie qui lui confie en 1828 une mission d'expertise sur les moteurs en usage dans les arsenaux et fonderie de l'État, une mission qui lui prend quatre années de travail méticuleux sur la question des frottements dans les machines sur laquelle aucune étude n'avait été entreprise dans le passé. Le mémoire qu'il rédige sur cette mission, remarquable par le souci de présentation pédagogique, est jugé par le Comité comme un « véritable cours de mécanique appliquée " et inséré au Mémorial de l'Artillerie, la revue savante du corps. Au contact de Poncelet, Morin qui est promu son adjoint en 1829, développe la partie instrumentale pour mener à bien ses expériences, en mettant au point divers appareils de mesure d'un type nouveau (chronomètres et dynamomètres à style) ${ }^{27}$. C'est en 1833 qu'il est

25 Claudine Fontanon, « Arthur Morin (1795-1880)... ») op. cit, pp. 2-6. 26 Bruno Belhoste, La formation d'une technocratie. L'École polytechnique et ses élèves de la Révolution au Second empire, Paris, Belin, 2003, pp. 351-354 ("Les préparationnaires"). 27 Arthur Morin « Mémoire sur divers appareils chronométriques et dynamométriques 1 , CR/AS, 1836, tome III, p. 22 ; " Un prix est décerné à Arthur Morin pour ce mémoire adressé au concours du prix de mécanique pour l'année 1836 ॥, CR/AS, tome VI, 1838, p. 260. et aussi « Mémoire sur deux appareils dynamométriques pour mesurer la force des moteurs animés ou les efforts de traction ainsi que les quantités sollicité par le Comité de l'artillerie pour former avec Piobert et Didion l'équipe de la Commission des principes du tir. Il y restera jusqu'en 1841, se chargeant au fil des années plus particulièrement des questions d'hydrodynamique qu'il avait abordées dans ses années de collaboration avec Poncelet. II mène en même temps une série d'expérimentations sur le tirage des voitures (1837-1841) à la demande du Comité et dans le cadre de la révision de la réglementation de la circulation routière. Il en découlera une polémique avec l'ingénieur des Ponts et Chaussées Jules Dupuit, polémique analysée dans ce dossier thématique par Konstantinos Chatzis.

Pour l'ensemble de ses travaux expérimentaux sur les moteurs hydrauliques, sur la question des frottements, pour ses expériences de balistique et d'hydrodynamique menées en collaboration avec Piobert et/ou Didion, enfin pour la réalisation des instruments de mesure nouveaux, le capitaine Morin rejoint son maître, Jean Victor Poncelet, à l'Académie de sciences (section mécanique) en 1843. En 1839, Poncelet avait également favorisé sa nomination à la chaire de mécanique appliquée fondée à sa demande au Conservatoire royal des arts et métiers. II en sera, on le sait, l'administrateur de 1849 à 1852 puis le directeur de 1854 à 1880, année de sa disparition. C'est au régime impérial auquel il adhère avec conviction que Morin doit sa promotion au grade de général de brigade en 1852 et de général de division en 1855. Morin consacrera l'essentiel de son temps au cours des décennies suivantes au Conservatoire dont il fera la renommée qui accompagnera l'institution pendant plus d'un siècle, ainsi qu'à la vie académique ${ }^{28}$.

Comme le soulignait Bruno Behoste dans son travail sur Poncelet, Piobert, Morin et Didion appartiennent à la catégorie des ingénieurs militaires qui, se considérant plus ingénieurs que militaires et qui, enseignant dans les écoles militaires, « entreprirent parallèlement à leur carrière d'officier une véritable carrière scientifique avec l'appui de leurs supérieurs $11^{29}$. C'est au début de la carrière scientifique d'Arthur Morin et en particulier à ses recherches hydrodynamiques, menées sur le terrain, dans la cadre de la Commission des principes du tir

de mouvement qu'ils développent "), Bulletin de la Société d'encouragement pour l'industrie nationale, $36^{\circ}$ année, mai 1837, pp 161-179.

28 Claudine Fontanon, "Morin, Arthur (1795-1880) ", Les professeurs du Conservatoire national des arts et métiers. Dictionnaire biographique, 1794-1955, Paris CNAM/INRP, 1994, vol. II, pp. 311-321.

29 B. Belhoste, « Jean Victor Poncelet... », op. cit., pp. 33-35. 
que nous nous intéresserons maintenant, un aspect de l'œuvre scientifique du savant officier d'artillerie que nous n'avions pas abordé dans nos travaux antérieurs.

\section{Les expériences d'hydrodynamique du capitaine Morin (1836-1841)}

Les expériences en plein air sur la résistance de l'eau à l'avancement des corps de différentes formes pourraient, comme Morin en fait lui-même la remarque " paraître étrangères au but du programme [de la commission des principes du tir]. Mais [ajoute-t-il], il a paru indispensable de s'intéresser à la résistance de l'eau, transition simple et naturelle entre les corps solides et les fluides élastiques $1^{30}$. Nous tenterons d'éclairer les circonstances qui ont permis à Arthur Morin, associé soit à Piobert soit à Didion de passer des questions de la résistance des projectiles à la pénétration de différents milieux (notamment à l'air) à celle de la résistance de l'eau à l'avancement des corps immergés puis des corps flottants pour aborder finalement la question du halage des bateaux, très éloignée de la balistique théorique et expérimentale explorée par Piobert et Didion dans le cadre du programme de la commission. Nous nous attacherons à caractériser la méthodologie imaginée par le « savant officier » pour mener ces expériences en plein air dans des conditions très éloignées des conditions d'expérimentation en laboratoire mais avec tous les attributs de la scienticité.

Les premières expériences de Morin sur la résistance de l'eau au mouvement des corps répondaient à la deuxième question du programme de la commission. Elles ont lieu à Metz sur la Moselle entre 1836 et 1838 avec le capitaine Didion. La seconde série d'expériences se dérouleront aux environs de Paris en 1839 et 1840 sur la Marne puis sur le canal de l'Ourq. Morin y mena seul les expériences sur le halage des bateaux.

Expériences sur les corps immergés dans l'eau Au départ, seule la loi de la résistance de l'air donnée par Newton devait être vérifiée par l'expérience dans le but de compléter les expériences antérieures menées par Hutton et Robins en Angleterre au XVIII siècle et par Borda en France dont les coefficients étaient toujours en usage vers $1830^{31}$. Mais avec les

30 SHD -2W 296: Vle rapport sur la loi de la résistance de l'eau au mouvement des corps abandonnés à l'action de la gravité au moyen d'un appareil chronométrique.

31 SHD-2W 296 : IIle rapport sur la résistance de l'air et de l'eau au mouvement des corps, 24 décembre 1838 rapport signé Piobert Morin et Didion : chap. II « Expériences sur la résistance de l'eau au mouvement des corps plans, progrès de l'expérimentation et les nouvelles exigences de l'artillerie ces coefficients sont dès lors considérés comme insuffisants. C'est dans cette optique que Piobert et Morin entreprennent à Metz en 1836 une étude sur la loi de la résistance de l'air par des tirs horizontaux avec des pendules balistiques conçus par Piobert et construits par Morin pour l'enregistrement de la valeur de la résistance de l'air à l'avancement et la pénétration des projectiles dans différents milieux aux vitesses balistiques (50 à $300 \mathrm{~m} / \mathrm{s}$ ) ${ }^{32}$. Puis les mêmes expériences sont exécutées dans l'eau pour des gros calibres et aux grandes vitesses, " quoi que ce genre de tir se présente rarement à la guerre mais présente un intérêt pour la science ». Pour les expérimentateurs il s'agit de "l'une des questions les plus importantes pour la pratique de l'artillerie et les plus intéressantes pour le progrès des sciences physiques et la recherche des lois de la résistance que les liquides et les gaz opposent au mouvement des projectiles et des corps de différentes formes aux petites et grandes vitesses $\|^{33}$. Les rapporteurs, Morin et Piobert, évoquent en introduction de leur récit d'expériences, « les très belles recherches entreprises à Metz depuis 1827 par Messieurs Poncelet et Lesbros sur les lois de l'écoulement de l'eau à travers les orifices "). Ceux-ci ayant fait établir sur la rive droite du grand bras de la Moselle un bassin de $1500 \mathrm{~m}^{3}$ fermé du côté de la rivière par une digue et par un barrage solide alors qu'en aval étaient construits deux bassins de jaugeage en maçonnerie. Morin et Piobert se propose d'y lancer des projectiles horizontalement et parallèlement à la surface de l'eau en traversant la paroi du barrage. Pour disposer les pièces d'artillerie, sont construits deux chevalets et une plateforme dont le sol est incliné à deux mètres environ en dessous du niveau habituel de l'eau. Les pièces d'artillerie sont pointées horizontalement de manière à frapper dans un mur de lattes posées sur le sol du bassin sur toute sa longueur. Pour tirer, « on levait la vanne en donnait le signal, le coup partait et le projectile pour pénétrer dans l'eau, n'avait à traverser qu'une solive mince qui altérait peu la vitesse ॥. Pour connaître leur point de chute (ou la portée horizontale du projectile), un vannage de lattes équidistantes de 0, 25 mètre en 0,25 mètre a été construit et les intervalles remplis de terre molle. Enfin, de cinq mètres en cinq mètres, ont été plantées des lattes verticales qui dépassent le niveau de l'eau et permettent pendant le tir

sphériques cylindriques ou coniques 11.

32 SHD- 2W 296, III rapport de la Commission des principes du tir (1835), "Pénétration des projectiles dans l'eau " (manuscr. ) chap. 3.

33 SHD-2W 296 III rapport, op. cit. 
d'observer « certains effet ॥. Immédiatement après le tir, on laisse écouler l'eau par une vanne du bassin et les résultats pouvaient être relevés quelques heures après. La procédure expérimentale consistait à faire varier chaque élément exerçant une influence sur la résistance à l'avancement du projectile (variation de l'aire du grand cercle du calibre (de huit, douze et vingt-quatre pouces), de son poids pour un même diamètre (de six à trente-neuf kilogrammes). Les observateurs sont placés sur la digue à côté du barrage pour observer les effets apparents. Ceux constatèrent un effet remarquable: la rupture des obus aux vitesses peu considérables de $356 \mathrm{~m} / \mathrm{s}$, fait singulier indiquant « l'énorme influence de la vitesse sur l'intensité de la résistance du milieu ॥. Le rapport se termine sur une description minutieuse de 13 pages du dispositif expérimental par Piobert et Morin.

C'est probablement ces expériences qui donnent à Morin l'idée d'entreprendre toujours à Metz en 1836 des séries d'expériences sur la résistance de l'eau au mouvement des corps de différentes formes ${ }^{34}$. Les corps qu'il soumet à l'expérience sont des plateaux minces remontant dans l'eau sous l'action d'un contre poids, des sphères pleines et creuses, des cylindres en fer de différents diamètres, des cônes terminant des cylindres, des cylindres terminés par des demi sphères et soumis à l'action de la gravité. Le dispositif expérimental est placé sur la Moselle en face à un déversoir où l'eau en surface est sans vitesse et la profondeur de cina mètres. Le mouvement vertical est produit par la gravité et par un lest pour augmenter la vitesse. La loi du mouvement est observée et déterminée par un chronomètre à style minutieusement décrit dans un rapport annexe ${ }^{35}$. Les résultats de ces observations sont que la résistance de l'eau croit si rapidement avec la vitesse que le mouvement devient très vite uniforme. L'expérimentateur procède ensuite au calcul de la résistance du fluide et à la recherche de la loi. Les conclusions sont les suivantes : la résistance de l'eau à l'avancement de corps de différentes formes se compose de deux termes, l'un indépendant de la vitesse et proportionnel à la surface movillée, l'autre proportionnel au carré de la vitesse. Le premier terme étant négligeable par rapport au second, la résistance est donnée par la formule: $R=K A V^{2}$, la valeur de $K$ étant déduite des expériences et $A$ correspondant à la projection du corps sur un plan perpendiculaire au sens du mouvement. Les résultats

34 SHD-2W 296 (1836) : Vle rapport à la Commission des principes du tir , chap. II : « Expériences sur la résistance de l'eau au mouvement des corps plans, sphériques cylindriques ou coniques $»$. 35 Archives SHD -VIe rapport ... op. cit , « Mémoire sur les appareils chronométriques $॥$. condensés dans un tableau permettent de conclure que les sphères sont les corps qui offrent le moins de résistance et s'appliquent aux projectiles et aux piles de ponts.

Mentionnons qu'au cours des expériences, l'appareil enregistreur ayant prouvé son imprécision en cas de variation de la vitesse de chute, on avait dû ajourner les expériences et chercher à énoncer les conditions pour que le poids moteur exerce un effort constant, ce qui amène à imaginer de faire varier la hauteur de l'installation du dispositif en fonction des vitesses recherchées. Cette manœuvre impliquait que l'appareil fût facilement transportable. Toutes les améliorations de l'appareil aussi infimes fussent-elles sont consignées dans le rapport annexe; par exemple le graissage des poulies, l'attention accordée au support des courbes (papier collé, fevilles de bois de placage ...) et à la fluidité de l'encre de Chine.

Vingt ans après l'exécution de ces expériences en plein air sur les corps de différentes formes, Morin devait résumer ce travail dans la seconde édition de ses Leçons de mécanique pratique tout en précisant que ces expériences n'avaient aucun intérêt industrie| ${ }^{36}$.

Les expériences sur les corps semi-immergés

De la résistance des corps immergés, Morin va s'intéresser à la résistance des corps semi-immergés comme les roves à palettes planes dont la principale application est alors la rove à aubes des bateaux à vapeur $^{37}$. Pour se rapprocher le plus possible des cas pratiques, les expériences ont été réalisées dans un canal en eau morte puis dans l'eau courante de la Moselle. Morin explique le choix des lieux : le bassin sur la rive droite de la Moselle est celui que Poncelet et Lesbros ont conçu et utilisé pour leur étude sur le débit de l'eau, de même que le canal en bois construit en aval du barrage, dont le débit est désormais connu.

Des expériences ont été effectuées en 1837 sur deux modèles de rove, dont la plus petite de 1 mètre de diamètre, reçoit des palettes planes en nombre variable (jusqu'à vingt) et de différentes longueur et largeur. L'arbre de la rove sert de trevil pour l'enroulement de la corde qui passe au sommet d'une chèvre à dix-sept mètres de hauteur supportant une caisse chargée de poids moteurs. On fait varier la

36 Arthur Morin, Leçons de mécanique pratique à l'usage des auditeurs du Conservatoire des arts et métiers et des sous officiers d'artillerie. « Notions fondamentales et données d'expériences 11, Paris, Hachette, 1855, Paris Hachette, pp. 363-403, chap : Résistance des fluides.

37 SHD-2W 296, Vle rapport de la commission des principes du tir : chap 1 «Expériences sur la résistance que l'eau oppose au mouvement des roves à palettes planes », 20 p. manuscr., tabl. 
profondeur d'immersion en élevant ou abaissant le niveau de l'eau ainsi que la vitesse de rotation de la roue jusqu'à $6 \mathrm{~m} / \mathrm{s}$ ainsi que le nombre des ailettes. Les expériences sont effectuées avec un seul élément variable.

La résistance de l'eau selon la surface immergée des palettes de la rove est enregistrée par une courbe avec un appareil enregistreur à style qui fait l'objet d'une description minutieuse en annexe du rapport. Les résultats obtenus selon la formule de la résistance $R=k^{\prime} A+K A V^{2}$ sont ensuite comparés à l'expression de la résistance donnée par Poncelet dans son cours des machines à l'École de Metz soit $R=0,934 \mathrm{~kg} / \mathrm{m}^{2}$. Les résultats des expériences montrent que la résistance opposée par l'eau au mouvement des palettes est proportionnelle à la surface de la palette moyennement immergée mais que la loi cesse d'être applicable à certaines vitesses de l'eau lorsque la résistance des palettes diminue et s'annule à certaines profondeurs d'immersion et vitesses de la rove. L'expérimentateur en tire la conclusion qu'il est nécessaire d'établir entre les profondeurs d'immersion des palettes, leur écartement et leur vitesse des rapports tels que l'eau ait toujours le temps de remplir le vide formé par la rotation des palettes et que pour chaque rove construite il y ait une limite de vitesse de marche convenant à son meilleur effet. Car la loi de proportionnalité de la résistance au carré de la vitesse est vérifié pour des vitesses de 1,66 $\mathrm{mS}$ à $1,9 \mathrm{~m} / \mathrm{s}$ lorsque l'écartement des palettes à la circonférence est comprise entre deux et trois fois leur profondeur d'immersion. Ce qui de l'avis de Morin est conforme aux cas pratiques.

Pour évaluer l'influence de la présence d'un bateau près de la rove, deux séries d'expériences furent menées avec un bateau placé à proximité de la rove et immergé de quantité égale à celle dont les aubes de la rove plongent dans l'eau, pour deux profondeurs d'immersion. Les résultats indiquaient que la résistance dans cette configuration diminuait en quantité très faible. Ces expériences sur les roues à ailettes sont consignées dans le $\mathrm{vl}^{\mathrm{e}}$ rapport à la commission des principes du tir le 24 décembre 1838 et signé par Morin, Piobert et Didion.

\section{Les expériences sur les corps flottants}

Seul, Morin va alors décider de poursuivre les recherches sur la résistance des corps flottants qui s'applique aux bateaux. II en donne un aperçu dans la réédition du premier volume de ses Leçons aux auditeurs du Conservatoire des arts et métiers consacré aux notions fondamentales de la mécanique appliquée ${ }^{38}$.

38 Arthur Morin, Leçons de mécanique pratique à l'usage des auditeurs du conservatoire des arts et métiers, vol. 1 : "Notions fondamentales », 2e éd.1855, pp. 363-395.
La description des expériences revêt les mêmes caractéristiques que précédemment. L'auteur est soucieux de détailler avec la plus grande précision les circonstances des essais, le dispositif expérimental utilisé à l'occasion de cette nouvelle série d'expériences, les résultats obtenus présentés sous forme de tableaux détaillés et les champs d'application possibles.

Les expériences ont été réalisées à Metz dans le fossé de la Courtine du fort Saint-Vincent dont les dimensions sont de trois cents mètres de long et trente mètres de large et 0,80 à 1,20 de profondeur. Un bateau à fond plat sert aux expériences; le mouvement lui est communiqué par une chèvre reliée à une corde s'enroulant sur deux tambours; les relevés de la vitesse se font avec un chronomètre à style et par un observateur installé à l'avant du bateau et équipé d'une montre chronomètre, celui-ci étant chargé de relever les temps de passage du bateau devant les divisions inscrites tous les dix mètres sur les murs du bastion. La vitesse ainsi déterminée, Morin examine l'inclinaison du bateau par l'intermédiaire de lattes et de tasseaux noircis et blanchis à la craie et pour des vitesses variant de $1,85 \mathrm{~m} / \mathrm{s}$ à $5 \mathrm{~m} / \mathrm{s}$.

Les résultats montrent que l'inclinaison augmente avec la vitesse et que l'onde solitaire produite par le bateau est observée en référence aux travaux publiés par Scott Russel. Morin s'appuie donc sur les travaux de l'expérimentateur écossais dont le mémoire vient d'être traduit par deux ingénieurs des Ponts et Chaussées, Emery et Mary sous le titre : "Recherches expérimentales sur la loi de certains phénomènes hydrodynamiques qui accompagnent le mouvement des corps flottants $\%$. Ce mémoire avait souligné un paradoxe : la résistance de l'eau était plus grande au petit trot qu'au galop. II mettait aussi en évidence le phénomène de l'onde solitaire et concluait à l'indépendance de sa vitesse par rapport à celle du bateau.

Les premières expériences de Morin doivent cependant être interrompues à Metz car au-delà d'un certain poids moteur communiqué par la chèvre au bateau, des accidents se produisaient. Morin décide alors d'utiliser des chevaux de halage pour tracter le bateau dans le sens contraire à son mouvement. Par ce dispositif, il obtient un parcours uniforme de cent quatre-vingts mètres au bout de cent vingt mètres de traction; les mesures sur l'inclinaison du bateau sont refaites à l'aide d'un tube en verre portant une échelle graduée et traversant le fond du bateau. Les premières conclusions auxquelles arrive Morin sont que l'onde solitaire est approximativement celle du bateau pour des vitesses de 1,50 à 5,5 mètres par 
L'onde solitaire a été découverte expérimentalement en 1834 par un ingénieur écossais : John ScottRussell. Alors qu'il observait le mouvement d'une péniche sur l'Union Canal qui relie Edimbourg à Glasgow, Scott-Russell remarqua un nouveau type d'onde se propageant à la surface du canal. II en fit la description dans les termes suivants:

« J'observais le mouvement d'un bateau qui était tiré rapidement le long d'un canal étroit par une paire de chevaux, quand soudain le bateau s'arrêta, mais il n'en fut pas de même pour la masse d'eau dans le canal qu'il avait mise en mouvement; elle s'accumula autour de la prove du bateau dans un état de violente agitation, puis soudainement l'abandonna, roula vers l'avant à grande vitesse, prenant la forme d'une grande élévation solitaire qui continua sa course le long du canal, apparemment sans changement de forme ou diminution de vitesse.

Je la suivis à cheval et la dépassai alors qu'elle roulait encore à la vitesse de 8 ou 9 miles à l'heure, préservant sa forme originale de 30 pieds de long et d'un pied à un pied et demi en hauteur. La hauteur diminua peu à peu et après une poursuite de un à deux miles, je la perdis dans les méandres du canal. Telle fut, dans le mois d'août 1834 ma rencontre fortuite avec ce magnifique et singulier phénomène ... ».

encadré 2 : John Scott-Russell, « Report on Waves », Proceeding of the Royal Society, Edimburg, Scotland, 1844, pp. 319-320.

seconde. Tous les résultats montrent que la résistance au halage des bateaux à fond plat ne suit dans aucun cas de la pratique la loi de proportionnalité à la surface du profil immergé et au carré de la vitesse même quand on tient compte de l'inclinaison du bateau en mouvement quelle que soit la vitesse. Ainsi la loi qui relie les données est complexe à établir et ne peut, selon l'expérimentateur, s'exprimer de manière simple. Quant à l'inclinaison, plus le bateau est long moins l'inclinaison augmente pour une même vitesse mais la résistance au frottement de l'eau sur les parois accroît la résistance à l'avancement. Il observe par ailleurs à l'arrière du bateau un phénomène qu'il désigne sous le vocable « d'effet de bouillonnement» (on parlerait aujourd'hui de cavitation) qui peut être diminué par une forme plus convenable de l'arrière du bateau (par une arête vive verticale). A partir de ces conclusions provisoires, Morin envisage bientôt d'améliorer la procédure d'expérience et de trouver d'autres conditions d'expérimentation.

Les expériences sur le halage des bateaux en région parisienne (1838-1841)

En 1837 alors que Morin travaille avec Piobert à la pénétration des projectiles dans différents milieux et à l'étude des corps immergés ou flottants, il apprend qu'un service de poste vient de s'ouvrir avec des bateaux rapides entre Meaux et Paris. II décide alors de poursuivre ses expériences sur le halage des bateaux avec le concours de la compagnie exploitante $^{39}$. Les conditions de l'expérience sont les

39 Archives SHD-2 W297 : VIIe rapport de la Commission des suivantes : le trajet entre Meaux et Paris est de onze lieues, les chevaux mettent $3 \mathrm{~h} 30$ à la descente et $4 \mathrm{~h}$ à la remonte avec un équipage de trois chevaux. Le bateau est spécialement conçu pour naviguer à des vitesses supérieures à $5 \mathrm{~m} / \mathrm{s}$; c'est un bateau est en tôle de 1,85 mètres de large du maitre couple, de 22, $70 \mathrm{~m}$ de long et 0,74 de haut hors d'œuvre à fond plat et à bordages verticaux. La prove est effilée et la poupe terminée par une arête verticale graduée tous les $0,60 \mathrm{~m}$; son poids à vide est de 4,3 tonnes pouvant accueillir 60 à 70 passagers plus quinze membres d'équipage. Pour établir des comparaisons, Morin décide par ailleurs d'utiliser des bateaux de différentes formes (nacelles, bateau d'équipage, de pontonniers, bateaux à rallonges). Le halage s'effectue à différentes vitesses avec des relais pour les chevaux tous les 2,75 lieues. Un système de décrochage de la corde de halage a été installé pour le passage des ponts et des obstacles. La résistance est enregistrée par un dynamomètre à style primé par la Société d'encouragement pour l'industrie nationale en $1837^{40}$.

principes du tir, Résistance de l'eau au halage des bateaux, manuscrit, 15 juillet 1839, signé Morin, Piobert, Didion.

40 Archives SHD-2 W296, Vle rapport de la commission des principes du tir ( 9 aout 1838) signé Morin Piobert et Didion «Description des appareils chronométriques à style et appareils dynamométriques propres à mesurer l'effort ou le travail développé par des moteurs ou par des organes de transmission du mouvement des machines, s'appliquant aux charrues et aux bateaux selon la définition du travail mécanique donnée par Poncelelet ». Ces appareils de mesure ont reçu le prix de la Société d'encouragement pour l'industrie nationale (comité des arts mécaniques) en 1838 : Mémoire sur deux appareils dynamométriques pour mesurer la force des moteurs animés ou les efforts de traction ainsi que les 
L'expérimentateur en donne la description suivante:

"L'appareil fournit sans le secours du calcul l'effort moyen ou variable exercé sur un bateau, un traineau, une voiture de wagon, une charrue pour tout corps soumis à un effort de traction. L'appareil doit être à la portée d'un simple ouvrier ou manœuvre. Ses indications doivent être indépendantes de la volonté de celui qui les emploie. Son prix doit être raisonnable pour des appareils mesurant des efforts de 100 à 600 kilogrammes 1.

Seize séries d'expériences sont exécutées en juillet 1838 pour effectuer les enregistrements, le relevé des courbes et le calcul des coefficients de la résistance à partir de la formule donnée par les théoriciens $s^{41}: R=k a(V+-V)^{2}$. Un soin particulier a été apporté à la construction du dynamomètre: Morin en avait définit les caractéristiques en 183642. Avec cet appareil, Morin précise le coefficient invariable de la définition de la résistance $R=10,43 \mathrm{~A}(\mathrm{~V}+-\mathrm{V})^{2}$.

Mais, dans le cours des expériences Morin rencontre des difficultés qu'il n'avait pas prévues : les chevaux de poste non dressés et réquisitionnés pour tirer le bateau ont du mal à avancer à une vitesse constante et se fatiguent vite en raison de l'instabilité des phénomènes hydrodynamiques, notamment celle de l'onde solitaire dans la résistance à l'avancement. Le postillon non préparé à des situations d'essais s'imaginait que le dynamomètre employé à mesurer la traction, était la cause des difficultés éprouvées par les chevaux et des accidents qui s'étaient multipliés. Rapidement, les essais ont dû être interrompus.

Morin devait en tirer les conclusions et déplacer le lieu de l'expérience dans l'arrondissement de Saint-Denis, sur le bief du canal Saint-Denis peu avant le pont de Flandre et modifier les conditions de l'expérience. Là, il utilise un bateau rapide construit

quantités de travail qu'ils développent ॥, Bulletin de la SEIN, mai 1837, pp. 161-179.

41 La résistance est dans un rapport constant avec le produit de l'aire (A) la plus grande du profil d'immersion par le carré de la somme des vitesses du bateau (V) et de l'eau ( $v$ ) (ou de la différence à la remonte (archives SHD-2W 297 : VIIe rapport à la Commission des principes du tir, op. cit.)

42 Leur sensibilité devait être proportionnelle à l'intensité des efforts à mesurer, le coefficient d'élasticité du métal devait être pris en compte ; la volonté de l'expérimentateur ne devait pas entrer dans le fonctionnement de l'appareil dont le maniement devait être simple, enfin on devait pouvoir l'adapter aux chemins de fer, au halage, au tirage des voitures et des charrues. sur le modèle de celui mis en service sur canal de Praisley en Ecosse ; le halage est fait avec des chevaux dressés fournis par le propriétaire du canal. Pour ne pas les fatiguer Morin décide de laisser l'onde solitaire passer devant le bateau et de ne reprendre l'allure que lorsque la surface de l'eau est redevenue calme. Le temps de parcours est aussi modifié et mesuré tous les cent mètres par un observateur muni d'un chronomètre d'une grande précision. Avec ce nouveau dispositif expérimental, l'objectif est de mesurer l'aire immergée de chaque profil de bateau à partir de l'inclinaison à chaque moment et pour toutes les vitesses. Seize séries d'expériences sont à nouveau conduites. Les résultats enregistrés par le dynamomètre sont traduits en tableaux de même que les variables sont calculées (celles de l'aire immergée). Les résultats font état du soulèvement du bateau en fonction de sa charge, peu considérable à faible charge mais avec l'abaissement du centre de gravité au delà de trois tonnes de charge additionnelle. On constate une inclinaison plus grande aux petites vitesses même régulières. Ceci amène Morin à la conclusion générale : l'instabilité des phénomènes hydrodynamiques ne permet pas de lier les phénomènes observés par une théorie et des formules assez simples pour être utiles à la navigation sur les canaux. Fort de ce constat réaliste, il préconise de réaliser à l'avenir des expériences sur petits modèles mus par des poids, dans une sorte de " bassin de carènes » qui ne sera finalement construit à Paris par les ingénieurs du Génie maritime qu'au début du XXe siècle ${ }^{43}$. Mais même dans ces nouvelles conditions, Morin doute qu'il soit possible de lier les faits observés par une théorie et des formules assez simples pour être utiles à la navigation.

Aussi, pour tirer bénéfice de ces expériences sur le halage des bateaux menées en région parisienne, Morin propose-t-il de représenter graphiquement les résultats d'expériences de manière à dégager une certaine continuité dans les phénomènes observés. Les courbes donneraient par interpolation graphique la valeur moyenne de l'effort correspondant à chaque vitesse pour un bateau donné. D'après cette méthode, Morin arrive à la conclusion que la résistance à l'avancement est à peu près dans un rapport constant avec le produit de l'aire maximale immergée du profil au repos par le carré de la somme des vitesses de l'eau et du bateau (ou sa différence à la remonte) pour la formule $R=K a(V+-v)^{2}$. Mais pour une même vitesse, la résistance peut varier

43 Serge Bindel, La détermination par essais sur modèles des qualités hydrodynamiques du navire, texte datylographié, s.d. DGA. 
du simple au double à cause de l'onde solitaire à l'avant du bateau. L'expérimentateur pointe alors les inexactitudes de la théorie de Scott-Russel et les conclusions erronées auxquelles il a abouti. Car Morin venait en effet de démontrer que la navigation était plus difficile sur les canaux larges que sur les canaux étroits et que la vitesse de l'onde solitaire n'était pas indépendante de celle du bateau mais lui est sensiblement égale pour un mouvement uniforme. Toutefois, quand la vitesse du bateau augmentait, celle de l'onde solitaire devenait autonome et engendrait des perturbations.

Toutes les données relatives à ces expériences en région parisienne sur le halage des bateaux sont consignées dans le vile rapport à la Commission des principes du tir et cosigné par un collectif d'ingénieurs (Mauduit, Piobert Pérignon, Baise...), bien que seul Morin ait effectué les séries d'expériences. Le rapport est adressé le 15 juillet 1839 au Comité de l'artillerie qui propose le 3 février 1840 son impression au Mémorial de l'artillerie. Le 22 mai, le ministre de la Guerre accepte sur sollicitation du Comité que le capitaine Morin complète les expériences sur la loi de la résistance des fluides et reçoive une dotation de mille huit cents francs. Mais il ne semble pas que ce dernier ait poursuivi ses expériences d'hydrodynamique appliquées à la navigation fluviale. Au même moment, le ministère de la Guerre lui propose d'examiner la question du tirage des voitures Le gouvernement s'occupait en effet depuis quelques années de fixer un règlement sur le chargement et la vitesse des véhicules routiers dans le but de limiter la dégradation des routes. Mais il ne disposait que d'observations très imprécises sur le sujet. Morin commença une série d'expériences à Metz ${ }^{44}$, puis fut autorisé à les poursuivre aux alentours du château de Vincennes sur la demande du ministère des travaux publics ${ }^{45}$. II en découla une longue polémique avec Jules Dupuit, ingénieur des Ponts et Chaussées, également mandaté pour cette mission par le ministère.

\section{Conclusion}

Quelles sont donc les caractéristiques des expériences menées par les ingénieurs de la Commission des principes du tir à la fin des années 1830 ? II faut souligner en premier lieu la dimension collective des ces recherches expérimentales menées sur le terrain,

44 Arthur Morin, Expériences sur le tirage des voitures faites en 1832 et 1839 à Metz, Metz, 1838, 103 p.

45 Arthur Morin, Expériences sur le tirage des voitures et sur les effets destructeurs qu'elles exercent sur les routes exécutées en 1837-1838 par ordre du ministère de la Guerre et en 18391841 par ordre du ministère des Travaux publics, Paris 1842. et la complémentarité des compétences associées et organisées par le biais des nominations du Comité de l'Artillerie (théoricien, expérimentateur, inventeur). ॥ est par ailleurs remarquable que l'équipe de recherche dispose d'une totale liberté d'organisation pour la conduite de ses travaux et dispose de moyens financiers importants pour les exécuter. Leurs résultats sont valorisés par le biais des responsabilités et des récompenses (rédaction de manuels, insertion des rapports au Mémorial de l'artillerie, promotion académique). II est vrai comme l'avait signalé Bruno Belhoste, que l'on se trouve dans un moment inédit de l'histoire où l'élite d'une génération née sous la Révolution formée à l'Ecole polytechnique et dotée d'un bagage scientifique élevé, mais aussi guidée par quelques figures de prove (notamment Poncelet) est portée par le défi économique de la compétition avec l'Angleterre. La construction d'une société nouvelle, sa modernisation par la science et l'innovation technique constitue un objectif mobilisateur pour ces jeunes ingénieurs militaires issus de la petite et moyenne bourgeoisie comme Morin et Piobert et parfois du peuple comme Didion.

En référence aux savants anglais qui les ont précédés sur le sujet de la balistique et de l'hydrodynamique, ces ingénieurs ont à cœur de construire une méthode expérimentale qui puisse fournir des données rationnelles et irréfutables. Cette démarche est en particulier illustrée par Morin qui multiplie le nombre des expériences dans des conditions similaires et avec une instrumentation spécialement conçue et réalisée pour atteindre une précision suffisante de manière à établir des coefficients représentatifs des phénomènes étudiés. \|l attache le même soin à décrire ses montages expérimentaux et instrumentaux à l'occasion des rapports qu'il adresse au Comité de l'artillerie, non seulement pour répondre à ses exigences méthodologiques de ce dernier mais pour laisser une trace précise des processus d'invention ou des procédures expérimentales jusqu'ici inédits. La plupart des rapports sont imprimés au Mémorial de l'artillerie qui couronne les meilleurs rapports des officiers de l'arme. On remarque le soutien actif que leur apporte leurs supérieurs en relayant leurs travaux au sein de l'Académie des sciences par l'organisation de concours (des sciences physiques et mathématiques) et la distribution de récompenses qui vont jusqu'à leur élection au sein de l'Académie des sciences (Piobert en 1840, Morin en 1843). C'est ainsi qu'à la suite de Poncelet et de Piobert, Morin conquiert un titre de savant à part entière et se consacre désormais à promouvoir l'expérimentation 
en mécanique appliquée aux activités économiques (agriculture industrie et transports). Sa notoriété devait rapidement franchir les limites de la sphère militaire. Ne participe-t-il pas en effet à l'exposition des produits de l'industrie nationale avec ses dynamomètres-enregistreurs en $1839^{46}$ pour lesquels il dépose un brevet l'année suivante. Confirmé dans son rôle d'expert en mécanique appliquée

46 Exposition des produits de l'industrie française en 1839. Rapport du jury central. "Dynamomètres ». Médaille d'or décernée à Arthur Morin, rue de l'Arcade Paris $9^{\circ}$. et d'expérimentateur hors pair par son élection à l'Académie des sciences, Morin aura aussi à cœur de transmettre son savoir accumulé dans ses années de recherche à la Commission des principes du tir, en insérant les résultats de ses expériences en plein air dans ses Leçons de mécanique à l'usage des auditeurs des cours du Conservatoire des arts et métiers ("Notions fondamentales " qui en seront à leur troisième édition 1860). 